\title{
Growth, optical characterization, and laser operation of a stoichiometric crystal $\mathrm{KYb}\left(\mathrm{WO}_{4}\right)_{2}$
}

\author{
M. C. Pujol, M. A. Bursukova,* F. Güell, X. Mateos, R. Solé, Jna. Gavaldà, M. Aguiló, J. Massons, and F. Díaz \\ Grup de Física i Cristal.lografia (FiCMA), Universitat Rovira i Virgili, 43005 Tarragona, Spain \\ P. Klopp, U. Griebner, and V. Petrov \\ Max-Born-Institute for Nonlinear Optics and Ultrafast Spectroscopy, D-12489 Berlin, Germany
}

(Received 9 October 2001; published 15 April 2002)

\begin{abstract}
We present our recent achievements in the growing and optical characterization of $\mathrm{KYb}\left(\mathrm{WO}_{4}\right)_{2}$ (hereafter $\mathrm{KYbW}$ ) crystals and demonstrate laser operation in this stoichiometric material. Single crystals of KYbW with optimal crystalline quality have been grown by the top-seeded-solution growth slow-cooling method. The optical anisotropy of this monoclinic crystal has been characterized, locating the tensor of the optical indicatrix and measuring the dispersion of the principal values of the refractive indices as well as the thermo-optic coefficients. Sellmeier equations have been constructed valid in the visible and near-IR spectral range. Raman scattering has been used to determine the phonon energies of KYbW and a simple physical model is applied for classification of the lattice vibration modes. Spectroscopic studies (absorption and emission measurements at room and low temperature) have been carried out in the spectral region near $1 \mu \mathrm{m}$ characteristic for the ytterbium transition. Energy positions of the Stark sublevels of the ground and the excited state manifolds have been determined and the vibronic substructure has been identified. The intrinsic lifetime of the upper laser level has been measured taking care to suppress the effect of reabsorption and the intrinsic quantum efficiency has been estimated. Lasing has been demonstrated near $1074 \mathrm{~nm}$ with $41 \%$ slope efficiency at room temperature using a $0.5 \mathrm{~mm}$ thin plate of KYbW. This laser material holds great promise for diode pumped high-power lasers, thin disk and waveguide designs as well as for ultrashort (ps/fs) pulse laser systems.
\end{abstract}

DOI: 10.1103/PhysRevB.65.165121 PACS number(s): 78.20.Ci, 42.55.Rz, 81.10.Dn, 78.30.-j

\section{INTRODUCTION}

In recent years the $\mathrm{Yb}^{3+}$ ion has been recognized as a dopant with interesting potential for diode-pumped solid state lasers in the $1 \mu \mathrm{m}$ region. ${ }^{1-3}$ InGaAs laser diodes can be used for pumping such lasers between 0.9 and $1.0 \mu \mathrm{m}$. $\mathrm{Yb}^{3+}$ doped materials are attractive as efficient active media because they possess several important advantages over the widely used $\mathrm{Nd}^{3+}$ : three to four times longer emission lifetime increasing the energy-storage time, roughly three times smaller Stokes shift between absorption and emission (laser quantum defect) reducing the thermal load, extremely simple two-level electronic structure eliminating the possibility for unwanted competitive processes as excited-state absorption and upconversion. In addition the laser efficiency is not degraded by concentration quenching and very high doping level is possible which can considerably reduce the requirements to the beam quality of the laser diodes used for pumping. We present in this work a laser host with $100 \% \mathrm{Yb}^{3+}$ doping, $\mathrm{KYb}\left(\mathrm{WO}_{4}\right)_{2}$ (hereafter $\mathrm{KYbW}$ ), i.e., a stoichiometric laser material, describe its growth, optical and relevant laser properties, and demonstrate laser operation.

$\mathrm{K} R E \mathrm{~W}(R E=\mathrm{Y}$ or $\mathrm{Gd})$ are well known host materials for doping with rare earth ions. The low-temperature phase of these compounds, monoclinic $\mathrm{KREW}$, possesses an important physical anisotropy that can be used in interesting optical applications. These tungstates are also characterized by large third-order nonlinear optical susceptibility being efficient Raman converters for ps/ns laser pulses. ${ }^{4}$

$\mathrm{K} R E \mathrm{~W}$ doped with lanthanide ions can incorporate very high concentrations approaching the stoichiometric structure [i.e., KErW, KHoW (Refs. 5-7)]. Stoichiometric laser mate- rials are advantageous in laser devices with thin disk or waveguide designs and are especially suited for diode laser pumping. As compared to YAG or glasses used as hosts for $\mathrm{Yb}^{3+}, \mathrm{K} R E \mathrm{~W}$ has the advantage of higher absorption cross section than YAG which decreases the minimum pump intensity necessary to achieve transparency in the quasi-twolevel system of ytterbium. ${ }^{89}$ Diode pumping of KREW: $\mathrm{Yb}$ with $R=\mathrm{Y}$ or $\mathrm{Gd}$ has been demonstrated between 965 and $980 \mathrm{~nm} .{ }^{10,11}$ The emission cross section of $\mathrm{KREW}$ doped with $\mathrm{Yb}$ is higher than in YAG or in glasses and the thermal conductivity has an intermediate position (it is four times better than in glasses). The laser quantum defect is less than in YAG. The emission linewidth of ytterbium in KREW with $R=\mathrm{Y}$ or $\mathrm{Gd}$ is broader than in YAG and comparable to that in glasses. ${ }^{2,3,12-14}$ This linewidth is interesting not only for potential tuning but mainly for the generation and amplification of short ( $\mathrm{ps}$ or fs) laser pulses. Mode-locking of diode pumped KGdW:Yb and KYW:Yb lasers has been demonstrated, ${ }^{15,16}$ and utilization of the crystal anisotropy for maximum gain bandwidth culminated in the generation of 71 fs pulses with KYW:Yb in $2001 .{ }^{17}$ Also regenerative amplification of fs pulses in KYW: $\mathrm{Yb}$ was demonstrated in 2001. ${ }^{18}$ Whereas femtosecond pulses can provide the ultimate peak powers, much higher average powers and optimum conditions for frequency conversion to other wavelengths can be realized with slightly longer pulses (1 ps or more for Raman conversion). We note here only that the highest average power directly produced by a mode-locked laser $(16.2 \mathrm{~W})$ has been achieved with a thin disk YAG:Yb laser. ${ }^{19}$ This laser concept for efficient diode laser pumping which is in particular relevant to stoichiometric laser materials with short absorption lengths has enormous potential for high- 
power $\mathrm{CW}$ operation in the $100 \mathrm{~W}$ regime and most recently the advantages of ytterbium doped KYW and KGdW over YAG have been demonstrated achieving a record of $60 \%$ of optical conversion efficiency. ${ }^{20}$

In the present work we describe first the growth procedure used for $\mathrm{KYbW}$ which is based on the top-seeded-solution growth (TSSG) slow-cooling method. The optical measurements include determination of the optical indicatrix, the dispersion of the refractive indices, the thermo-optic coefficients, and the construction of a set of Sellmeier equations in the visible and infrared. The phonon energies are determined by Raman spectroscopy. The electronic substructure of the two ytterbium manifolds is resolved by room and low temperature absorption and emission studies with polarized light and the vibronic features observed in the absorption spectra are explained. The fluorescence lifetime is measured taking care to avoid reabsorption and internal reflections. Lasing near $1074 \mathrm{~nm}$ is demonstrated with a tunable Ti:sapphire pump source using a thin $(0.5 \mathrm{~mm})$ plate of $\mathrm{KYbW}$ at room temperature without special cooling. Quasi-CW laser operation with maximum conversion efficiency of $25 \%$ (slope efficiency of $41 \%$ ) has been achieved for pumping wavelengths of 963 and $922 \mathrm{~nm}$.

\section{CRYSTAL GROWTH AND STRUCTURE}

KYbW single crystals have been grown by the TSSG slow-cooling method. $\mathrm{K}_{2} \mathrm{~W}_{2} \mathrm{O}_{7}$ was chosen as a solvent because it does not introduce impurity ions and because its melting point is relatively low. The growing technique used was similar to that described in more detail by us in previous works. $^{21,22}$

We grew KYbW starting with a binary solution composition of $11.5 \mathrm{~mol} \%$ solute $/ 88.5 \mathrm{~mol} \%$ solvent. Platinum conical crucibles with a volume of $25 \mathrm{~cm}^{3}$ were used to prepare $50 \mathrm{~g}$ of solution using $\mathrm{K}_{2} \mathrm{CO}_{3}, \mathrm{Yb}_{2} \mathrm{O}_{3}$, and $\mathrm{WO}_{3}$ reagents from Aldrich and Fluka (99.9\% purity). The solution was homogenized by fixing the temperature at about 50 $\mathrm{K}$ above the expected saturation temperature $\left(T_{\text {sat }}\right.$, determined from the solubility curves ${ }^{23}$ ) for $5-6 \mathrm{~h} . T_{\text {sat }}$ was estimated with an accuracy of $0.1 \mathrm{~K}$, by observing the growth/ dissolution of a KYbW seed placed in the center of the free surface of the solution. The saturation temperature at the particular solute/solvent ratio used was $1170-1175 \mathrm{~K}$. Previous studies of KGdW: $\mathrm{Ln}^{3+}$ crystal growth indicated that parallelepipedic $\boldsymbol{b}$-oriented seeds improve the crystal growth rate and produce inclusion-free crystals.

The axial thermal gradient was $1 \mathrm{~K} / \mathrm{cm}$, the bottom being hotter than the surface of the solution. The radial thermal gradient was also $1 \mathrm{~K} / \mathrm{cm}$, and the crucible wall was hotter than the center of the solution. The slow-cooling rate was 0.1 $\mathrm{K} / \mathrm{h}$ for $15-20 \mathrm{~K}$. Crystal rotation, without pulling, was kept constant at $40 \mathrm{rpm}$. After four days of growth, single crystals were removed slowly from the solution and cooled to room temperature at a rate of $15 \mathrm{~K} / \mathrm{h}$ in order to minimize thermal shocks. Figure 1(a) shows a KYbW single crystal grown by this method.

Klevtsov and Kozeeva (1969) (Ref. 5) determined a KYbW phase transition temperature of $1303 \mathrm{~K}$ and a melting

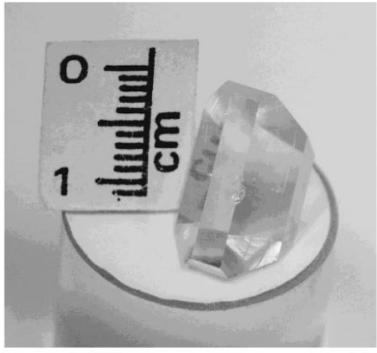

a)

b)

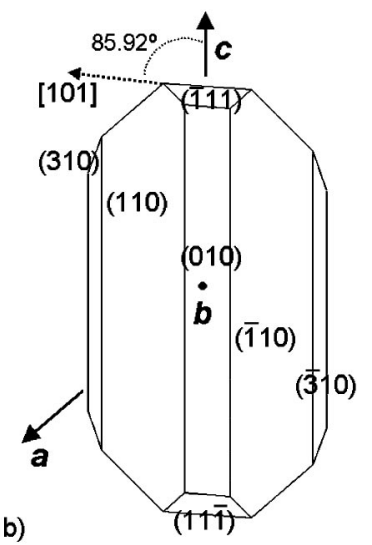

FIG. 1. (a) Photograph of a KYbW single crystal grown in the $\boldsymbol{b}$ direction. (b) A morphological sketch of KYbW.

temperature of $1318 \mathrm{~K}$. They obtained the unit cell parameters for the monoclinic $\mathrm{KYbW}$ phase from a $\mathrm{x}$-ray powder pattern and optical goniometric measurements but did not indicate the space group.

To improve their results we refined the crystal structure of monoclinic KYbW crystals at room temperature by using single crystal x-ray diffraction data. Single crystal diffraction was carried out on spherical crystalline samples and the structure was resolved by Patterson synthesis using the SHELXS97 computer program ${ }^{24}$ and refined by a fullmatrix least-squares method using the SHELXL97 computer program. ${ }^{25}$ Details of the unit cell structure and the interionic distances will be published elsewhere. ${ }^{23}$ The unit cell parameters are $a=10.590(4) \AA, b=10.290(6) \AA, c=7.478(2) \AA$, $\beta=130.70(2)^{\circ}$, and $Z=4$, with the space group $C 2 / c$.

The general Wyckoff position in KYbW is occupied by a distorted tetrahedron $\left[\mathrm{WO}_{4}\right]^{2-}$. Two oxygens from a neighboring tetrahedron are situated very close to it leading to a coordination figure of the tungstate anion of a distorted octahedron $\mathrm{WO}_{6}$. The electronic charge of the two "extra" oxygens extends towards the neighbor $\mathrm{W}^{6+}$, so that they do not contribute to the polarizability of the considered $\left[\mathrm{WO}_{4}\right]^{2-}$ molecular anion. Two $\mathrm{WO}_{6}$ octahedra are joined by sharing edges $\mathrm{O} \cdots \mathrm{O}$ (double oxygen bridge) and form a unit of two distorted octahedra $\left(\mathrm{W}_{2} \mathrm{O}_{10}\right)$. These units form a double chain along the crystallographic $c$ axis by sharing vertex $\mathrm{O}$.

The ytterbium is eight coordinated by oxygen atoms, forming a square antiprism. These polyhedra form a single chain in the [101] direction. The distance $\mathrm{Yb}-\mathrm{Yb}$ in the same chain is $4.049(2) \AA$ and from different chains it is 6.721(2) A. These separations are very important for the interaction between ytterbium ions (e.g., cooperative effects).

The alkali cation $\mathrm{K}^{+}$is twelve-coordinated by oxygens, forming a distorted icosahedron. These polyhedra form a bidimensional layer made up of chains that share edges in the [101] and [110] directions.

$\mathrm{KYbW}$ has a crystalline habit that is formed by the $\{110\},\{111\},\{010\}$, and $\{310\}$ faces. The first three faces are 


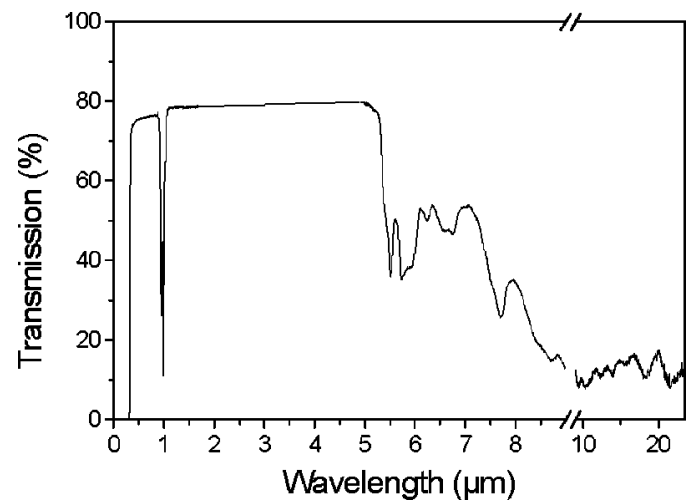

FIG. 2. Optical window of $\mathrm{KYbW}$ at room temperature measured with unpolarized light and a $0.2 \mathrm{~mm}$ thin sample of $\mathrm{KYbW}$ cut parallel to the $\boldsymbol{a}-\boldsymbol{c}$ plane. For better representation the curve is interrupted at $9 \mu \mathrm{m}$ to change the abscissa scale.

more developed than the last. In all grown crystals, the dimension along the $c$ crystallographic axis is approximately two times larger than the other two dimensions. Also, the $\boldsymbol{c}$ axis and the [101] direction of the crystals grown appear as natural edges. The [101] direction is located at $85.92^{\circ}$ from the $\boldsymbol{c}$ axis rotating anticlockwise around the $\boldsymbol{b}$ crystallographic axis, whose positive end is directed towards the observer [see the morphology sketch of KYbW shown in Fig. $1(\mathrm{~b})]$.

\section{TENSOR OF THE REFRACTIVE INDICES AND TRANSPARENCY WINDOW}

The measured transmission of KYbW for a sample thickness of $0.2 \mathrm{~mm}$ is shown in Fig. 2. The UV cutoff wavelength (zero level) is near $300 \mathrm{~nm}\left(33333 \mathrm{~cm}^{-1}\right)$ and the clear transparency for this thickness is roughly between 350 and $5000 \mathrm{~nm}$. The flat transparency region is narrowed to approximately 500-4700 $\mathrm{nm}$ when sample thickness above 1 $\mathrm{mm}$ is used. The absorption band near $980 \mathrm{~nm}$ will be analyzed in Sec. V.

The low-temperature phase of KYbW belongs to the $2 / m$ crystallographic point group, hence it is a biaxial crystal with inversion center. The three orthogonal principal crystallooptic axes are labeled $N_{g}, N_{m}$, and $N_{p}$ (or alternatively $z, y$, and $x$ ). In monoclinic crystals, one of the principal axes (in this case $N_{p}$ ) is parallel to the $C_{2}$ symmetry axis which coincides with the crystallographic $\boldsymbol{b}$ axis, thus, the other two principal axes (in this case $N_{g}$ and $N_{m}$ ) lie in the $\boldsymbol{a}$ - $\boldsymbol{c}$ plane because the $\boldsymbol{b}$ axis is orthogonal to this plane. The determination of the angle between the $c$ axis and the $N_{g}$ (or $N_{m}$ ) principal axis in the $\boldsymbol{a}-\boldsymbol{c}$ plane locates the orientation of the optical indicatrix of $\mathrm{KYbW}$.

The orientation of the principal crystallo-optic axes was determined at $\lambda=632.8 \mathrm{~nm}$ using two crossed Glan-Taylor polarizers. With the crystal between them we found that the principal axis $N_{g}$, is located at $\kappa=19.0^{\circ}$ with respect to the $c$ crystallographic axis where the angle of rotation $\kappa$ is measured in the clockwise direction when the positive end of the $\boldsymbol{b}$ axis is pointing towards the observer. $N_{m}$ is rotated at $59.7^{\circ}$ with respect to the $\boldsymbol{a}$ crystallographic axis in the clockwise

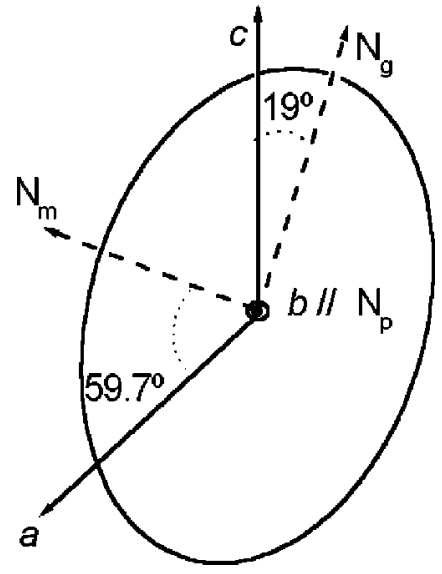

FIG. 3. Optical ellipsoid of $\mathrm{KYbW}$ at room temperature.

direction. Figure 3 shows the optical ellipsoid of KYbW.

We determined the dispersion of the three refractive indices labeled $n_{g}>n_{m}>n_{p}$ (or alternatively $n_{z}>n_{y}>n_{x}$ ) and measured the thermo-optic coefficients. The dispersion of the principal values of the refractive indices was measured between 0.45 and $1.5 \mu \mathrm{m}$ by the minimum deviation method with a semiprism as described in more detail in Ref. 26. Two prisms cut in different principal planes were used that allow one to obtain $n_{g}, n_{m}$, and $n_{p}$ (one of them twice) with an accuracy of $5 \times 10^{-4}$.

Figure 4 shows the dispersion of the refractive indices in the visible and near-IR spectral regions. As can be seen $\mathrm{KYbW}$ possesses significant birefringence and the three refractive indices are nearly equidistant. The latter is expressed in the angle $2 V_{z}$ between the two optic axes ${ }^{27}$ in the $N_{p}-N_{g}$ plane which is close to $90^{\circ}\left(2 V_{z}=100.6^{\circ}\right.$ determined from $\sin V_{z}=n_{g}\left(n_{m}^{2}-n_{p}^{2}\right)^{1 / 2} / n_{m}\left(n_{g}^{2}-n_{p}^{2}\right)^{1 / 2}$ at $\left.\lambda=632.8 \mathrm{~nm}\right)$. Since $2 V_{z}$ is larger than $90^{\circ}$ it follows that $\mathrm{KYbW}$ is an optically negative biaxial crystal.

We fitted the data points in Fig. 4 using one UV pole and an IR correction term with a Sellmeier equation of the form $n^{2}=A+B /[1-(C / \lambda)]^{2}-D \lambda^{2}$. Table I summarizes the Sellmeier coefficients obtained for $\mathrm{KYbW}$ at room temperature. We studied the behavior of the refractive indices with temperature in the $293-473 \mathrm{~K}$ range using a microfurnace with temperature control and taking care to homogeneously heat

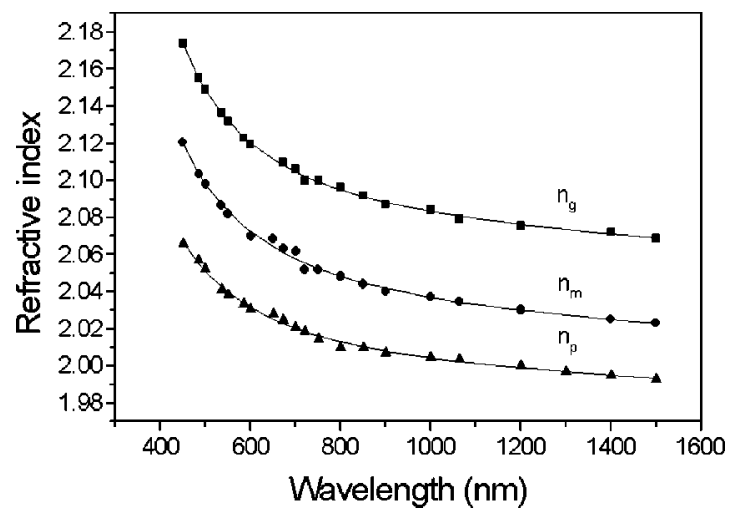

FIG. 4. Dispersion of the principal refractive indices of KYbW at room temperature. 
TABLE I. Room-temperature Sellmeier coefficients and thermooptic coefficients of KYbW.

\begin{tabular}{lccccc}
\hline \hline $\begin{array}{l}\text { Principal } \\
\text { refractive index }\end{array}$ & $\mathrm{A}$ & $\mathrm{B}$ & $\begin{array}{c}\mathrm{C} \\
\mu \mathrm{m}\end{array}$ & $\begin{array}{c}\mathrm{D} \\
\mu \mathrm{m}^{-2}\end{array}$ & $\begin{array}{c}\partial n / \partial T \\
\mathrm{~K}^{-1}\end{array}$ \\
\hline$n_{g}$ & 3.28412 & 0.9921 & 0.25426 & 0.01936 & $3.1 \times 10^{-5}$ \\
$n_{m}$ & 3.17884 & 0.91624 & 0.25087 & 0.00485 & $3.1 \times 10^{-5}$ \\
$n_{p}$ & 3.06172 & 0.88655 & 0.23858 & 0.02286 & $7.3 \times 10^{-5}$ \\
\hline \hline
\end{tabular}

the samples. The thermo-optic coefficients estimated at $\lambda$ $=632.8 \mathrm{~nm}$ are also included in Table I.

In monoclinic crystals also two of the principal crystallooptic axes (those lying in the $\boldsymbol{a}-\boldsymbol{c}$ plane) are subjected to dispersion ${ }^{27}$ and rotate around the $\boldsymbol{b}$ axis when changing the wavelength which can be characterized by the derivative $(\partial \kappa / \partial \lambda)$. A similar rotation of these two axes in the $\boldsymbol{a}$-c plane occurs also when changing the temperature, which can be accounted for by the derivative $(\partial \kappa / \partial T)$. We tried to estimate these two derivatives in KYbW using the methodology described above for the characterization of the optical ellipsoid, however, in the mentioned wavelength $(0.45-1.5 \mu \mathrm{m})$ and temperature $(293-473 \mathrm{~K})$ ranges both effects turned out to have magnitudes smaller than the error of the measurement.

\section{RAMAN SPECTROSCOPY}

The intermanifold electron transitions of the $\mathrm{Yb}^{3+}$ ion are strongly coupled with the lattice vibrations. The knowledge of the phonon frequencies is important for the interpretation of the substructure in the absorption and fluorescence spectra, and for the identification of the mid-IR cuttoff edge. Furthermore, spontaneous Raman scattering data could be used to evaluate the feasibility of $\mathrm{KYbW}$ as a stimulated Raman scattering (SRS) converter.

Polarized Raman scattering spectra for KYbW have been previously recorded and analyzed. ${ }^{28,29}$ For labeling and assignment of the fundamental modes a model was proposed, based on the consideration of $\mathrm{W}_{2} \mathrm{O}_{8}$ dimers plus weak W-O-W perturbations, resulting in centrosymmetric $\mathrm{W}_{2} \mathrm{O}_{10}$ clusters. The modes were classified by the irreducible representations of the $C_{i}$ group, additionally correlated with the $C_{2 h}$ crystal symmetry.

Here we present a simpler and physically more intuitive model for classification and labeling of the crystal modes, following an idea developed for scheelite-type tungstates and molybdates. ${ }^{30}$ In addition, our measurements reveal some differences in the phonon energies as compared to the values previously tabulated for $\mathrm{KYbW}^{28,29}$

$\mathrm{KYbW}$ exhibits chemical bonding typical of both ionic and covalent crystals. The vibrational frequencies of the molecular anions are known to be characteristic, i.e., they do not change significantly with the environmental perturbations. This suggests weak coupling between the $\left[\mathrm{WO}_{4}\right]^{2-}$ molecular anion and the rest of the crystal lattice. A very good and useful approximation in such a case is to divide the crystal vibrations into internal (for which the center of the mass of $\left[\mathrm{WO}_{4}\right]^{2-}$ does not move) and external (for which the
$\left[\mathrm{WO}_{4}\right]^{2-}$ ion is considered as a rigid unit).

One possible approach for the labeling of the internal modes of $\left[\mathrm{WO}_{4}\right]^{2-}$ is to start with the highest symmetry $T_{d}$ (the one the free $\left[\mathrm{WO}_{4}\right]^{2-}$ anions possess) represented by four irreducible representations $A_{1}+E+2 F_{2}$, then move to the lowest symmetry of $\left[\mathrm{WO}_{4}\right]^{2-}$ in $\mathrm{KYbW}\left(C_{1}=9 \mathrm{~A}\right)$, and finally consider the removal of the degeneracy of the $E$ and $F_{2}$ irreducible representations as a "splitting" induced by the crystal asymmetry.

The standard notation for the four fundamental mode frequencies of molecules of the type $X Y_{4}$ is $\nu_{1}\left(A_{1}\right), \nu_{2}(E)$, $\nu_{3}\left(F_{2}\right)$, and $\nu_{4}\left(F_{2}\right)$. To express the splitting of the last three modes, we add superscripts - and + for the doublet $\nu_{2}$, and ,- 0 , and + for the triplets $\nu_{3}$ and $\nu_{4}$ with,- 0 , and + in order of increasing energy. Omitting the designation of the irreducible representation in the brackets we denote the nine fundamental internal modes for simplicity as $\nu_{1}, \nu_{2}^{-}, \nu_{2}^{+}$, $\nu_{3}^{-}, \nu_{3}^{0}, \nu_{3}^{+}, \nu_{4}^{-}, \nu_{4}^{0}$, and $\nu_{4}^{+}$. Note that as a consequence of the $C_{1}$ symmetry interactions between all of these nine modes are allowed.

To justify the above mentioned approach we have to verify that the center of gravity for the four fundamental mode frequencies for $T_{d}$ symmetry $\nu_{1}$ to $\nu_{4}$ remains sufficiently stable in the course of the splitting when moving to lower symmetry. It is known that in aqueous solutions the $\left[\mathrm{WO}_{4}\right]^{2-}$ anion forms a regular tetrahedron. ${ }^{31}$ An unambiguous interpretation of the Raman spectra was presented in this early work, with three centers of gravity: the $931 \mathrm{~cm}^{-1}$ line was assigned to $\nu_{1}$, the $833 \mathrm{~cm}^{-1}$ diffuse band was assigned to $\nu_{3}$, and the $324 \mathrm{~cm}^{-1}$ broad band was assigned to both $\nu_{2}$, and $\nu_{4}$. In scheelite $\left(\mathrm{CaWO}_{4}\right)$ the broad band was resolved with gravity centers of $336 \mathrm{~cm}^{-1}$ for $\nu_{2}$ and $405 \mathrm{~cm}^{-1}$ for $\nu_{4} \cdot{ }^{30}$ The centers of gravity for the other two fundamentals reported for scheelite were at $912 \mathrm{~cm}^{-1}$ for $\nu_{1}$ and at $817 \mathrm{~cm}^{-1}$ for $\nu_{3} \cdot{ }^{30}$ Thus, reduction of the symmetry from $T_{d}$ to $S_{4}$ (the $\left[\mathrm{WO}_{4}\right]^{2-}$ site symmetry in scheelite) does not significantly modify the center of gravity for $\nu_{1}, \nu_{2}$, and $\nu_{3}$, but increases the center of gravity for $\nu_{4}$ by roughly $80 \mathrm{~cm}^{-1}$. Therefore, for KYbW, where the symmetry of the anion is additionally lowered to $C_{1}$ (no symmetry), one could expect preservation of the center of gravity for $\nu_{1}, \nu_{2}$, and $\nu_{3}$, and possible additional increase for the center of gravity for $\nu_{4}$.

The $2.65 \mathrm{~mm}$ thick sample of $\mathrm{KYbW}$ available for the Raman scattering measurements was cut and polished for propagation along the $N_{m}$ principal axis. The experimental setup comprised a Jobin-Yvon T64000 spectrometer with excitation in the visible by a $\mathrm{CW}$ argon laser (Coherent INNOVA 300, $\lambda=514 \mathrm{~nm}$ ). Behind the triplemonochromator $(1800 \mathrm{~g} / \mathrm{mm})$ the light was detected by a two-dimensional CCD matrix cooled with liquid $\mathrm{N}_{2}$. A premonochromator eliminated the plasma discharge lines of the argon laser. A microscope (Olympus BH2) with high resolution was used to locate the laser spot in the sample. The laser power incident on the sample was about $60 \mathrm{~mW}$. A backward scattering scheme was chosen in order to increase the signal to noise ratio.

Figure 5 shows the Raman spectra recorded at room tem- 


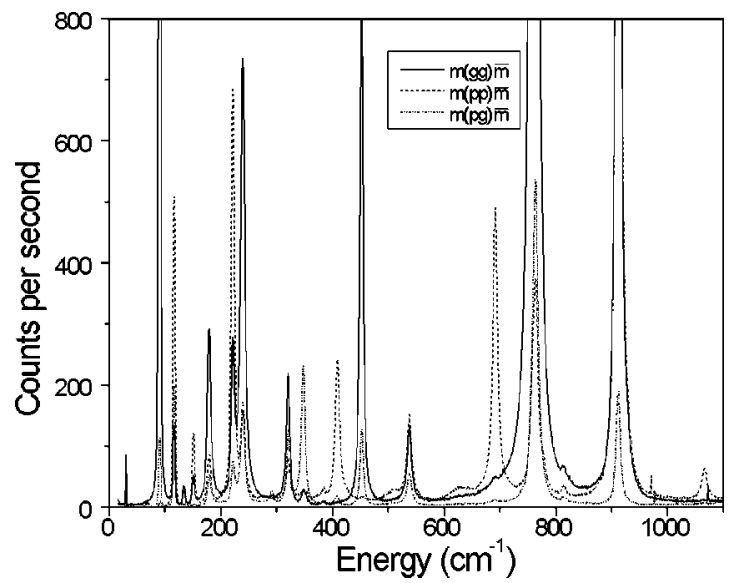

FIG. 5. Raman spectra of KYbW recorded at room temperature.

perature in the frequency range 0 to $1100 \mathrm{~cm}^{-1}$. The polarized spectra belong to the $m[g g] \bar{m}, m[p p] \bar{m}, m[p g] \bar{m}$, and $m[g p] \bar{m}$ (not shown) scattering configurations. The last two must be equivalent and both were recorded basically in order to confirm the sample orientation. As can be seen from the figure the doublet $\nu_{2}$ is split but its center of gravity is almost unchanged (339 $\mathrm{cm}^{-1}$ ). The triplet $\nu_{4}$ is additionally shifted (with respect to $\mathrm{CaWO}_{4}$ ) by about $50 \mathrm{~cm}^{-1}$ as could be expected. A shift of almost the same magnitude is observed for the center of gravity for the $\nu_{3}$ triplet, too. Note, however, that a similar shift at $\nu_{3}$ in the same direction has been recently observed in $\mathrm{NaY}\left(\mathrm{WO}_{4}\right)_{2}$ which has scheelite structure. $^{32}$

The two strongest peaks appear near 763 and $911 \mathrm{~cm}^{-1}$. Their large cross section makes $\mathrm{KYbW}$ attractive for SRS applications similarly to the analogous KYW and KGdW and especially interesting for the picosecond regime because of the relatively large linewidths (of the order of $10 \mathrm{~cm}^{-1}$ corresponding to a phase relaxation time of $1 \mathrm{ps}) .{ }^{4}$ Note that simultaneous laser operation and SRS to the first Stokes has already been demonstrated with KGdW:Yb. ${ }^{33}$ The weak lines at 1065,627 , and $150 \mathrm{~cm}^{-1}$ can be assigned to combination frequencies involving $\nu_{1}$ and $\nu_{3}^{0}$ as previously reported in SRS experiments. ${ }^{34}$

Table II summarizes the principal modes of $\mathrm{KYbW}$ as derived from our measurements and their assignment. The assignment of the external modes is known from $\mathrm{CaWO}_{4}$ and they are included in the table with their original notations. ${ }^{30}$ We note, however, that for the unambiguous assignment of all components of the tensor of the Raman polarizability six independent spectral measurements are necessary.

Some of the Raman lines identified in Table II can be observed in the mid-IR part of the transmission curve in Fig. 2. Thus, e.g., we could identify $\nu_{4}^{0}$ at $460 \mathrm{~cm}^{-1}, \nu_{4}^{+}$at $538 \mathrm{~cm}^{-1}$, and $2 \nu_{1}-\nu_{3}^{0}$ at $1068 \mathrm{~cm}^{-1}$ there. The strongest absorption lines in Fig. 2 appear at 1816 and $1300 \mathrm{~cm}^{-1}$ and we assign them to $2 \nu_{1}$ and $\nu_{1}+\nu_{4}^{-}$, respectively. Therefore we conclude that the mid-IR cutoff edge of $\mathrm{KYbW}$ is due to two-phonon $\left(2 \nu_{1}\right)$ absorption.
TABLE II. Vibrational frequencies for KYbW at room temperature. By "?" we indicated modes that could be identified as combination frequencies but are very weak in Fig. 5.

\begin{tabular}{cccc}
\hline \hline \multicolumn{2}{c}{ External modes } & Internal Modes \\
$\nu\left(\mathrm{cm}^{-1}\right)$ & Assigment & $\nu\left(\mathrm{cm}^{-1}\right)$ & Assigment \\
\hline 29 & unidentified & 320 & $\nu_{2}^{-}$ \\
68 & $?\left(\nu_{3}^{0} \nu_{3}^{-}\right)$ & 347 & $\nu_{2}^{+}$ \\
90 & $B_{g}$ & 381 & unidentified \\
115 & $E_{g}$ & 408 & $\nu_{4}^{-}$ \\
133 & unidentified $/ E_{u}$ & 452 & $\nu_{4}^{0}$ \\
150 & $E_{u} / \nu_{1}-\nu_{3}^{0}$ & 505 & $?\left(\nu_{1}^{-} \nu_{4}^{-}\right)$ \\
178 & $A_{u}$ & 537 & $\nu_{4}^{+}$ \\
221 & $E_{g}$ & 627 & $2 \nu_{3}^{0} \nu_{1}$ \\
239 & $B_{g}$ & 691 & $\nu_{3}^{-}$ \\
291 & unidentified & 763 & $\nu_{3}^{0}$ \\
& & 813 & $\nu_{3}^{+}$ \\
& & 911 & $\nu_{1}$ \\
& & 1065 & $2 \nu_{1}^{-} \nu_{3}^{0}$ \\
\hline
\end{tabular}

\section{YTTERBIUM SPECTROSCOPY}

\section{A. Absorption spectra}

We studied the polarized optical absorption of KYbW between room temperature and $6 \mathrm{~K}$ using a Cary Varian 500 spectrophotometer. Sample cooling for the low temperature measurements was accomplished by an Oxford Instruments cryostat (SU 12 model) with helium gas flow. KYbW has a clear absorption band between 12000 and $9100 \mathrm{~cm}^{-1}$ (820-1100 nm, see Fig. 2); this band is associated with the ytterbium transition ${ }^{2} F_{7 / 2} \rightarrow{ }^{2} F_{5 / 2}$. In Fig. 6 we show the room temperature absorption coefficient of this transition, measured for all three polarizations with a spectral resolution of $1 \mathrm{~nm}$. Sufficient signal to noise ratio in the whole spectral range presented could be achieved by sewing curves measured with more than one sample $(0.2$ and $3 \mathrm{~mm}$ thick for $E \| N_{p}, 0.2$ and $3.3 \mathrm{~mm}$ for $E \| N_{g}$, and $0.08,0.2$, and 3.3 mm thick for $\left.E \| N_{m}\right)$. Note that the absorption coefficient is strongly dependent on the light polarization and is minimal for $E \| N_{g}$. The room temperature absorption is characterized

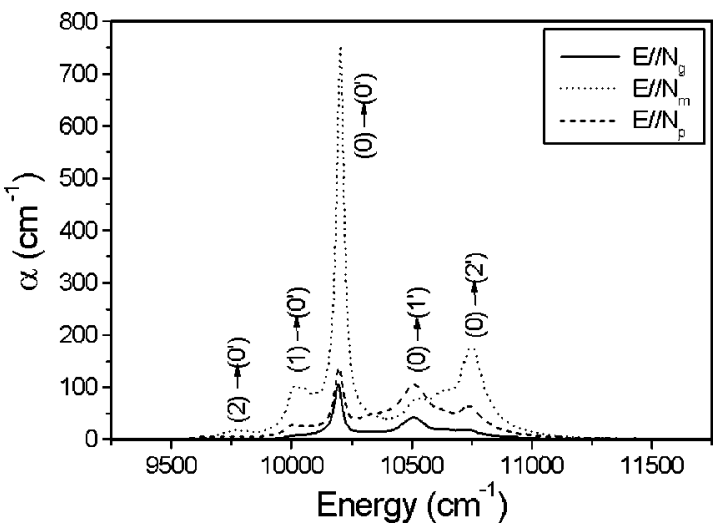

FIG. 6. Polarized optical absorption of KYbW at room temperature. 


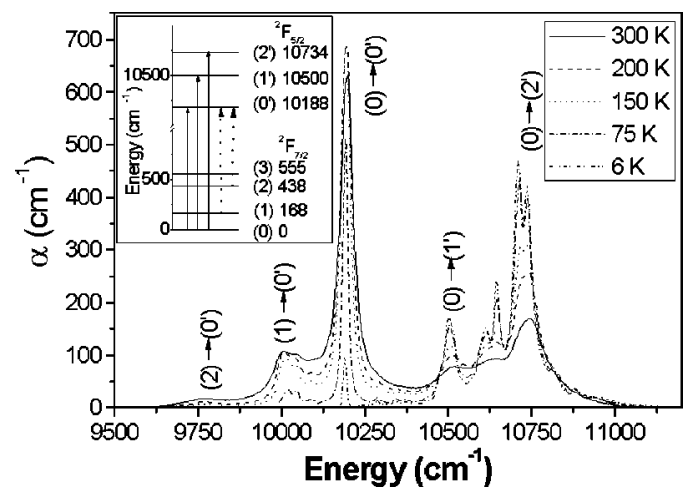

FIG. 7. Temperature evolution of the optical absorption of $\mathrm{KYbW}$ for $E \| N_{m}$.

by three main peaks which are centered at 10195,10518 , and $10742 \mathrm{~cm}^{-1}$ for $E \| E_{m}$, and two less intensive peaks near 10020 and $9770 \mathrm{~cm}^{-1}$ (the designation of the transitions can be seen in Fig. 7).

The maximum absorption cross sections at $981 \mathrm{~nm}$ (zero line) calculated with the $\mathrm{Yb}^{3+}$ concentration of 6.4 $\times 10^{21} \mathrm{~cm}^{-3}$ amount to $1.17 \times 10^{-19} \mathrm{~cm}^{2}$ for $E \| N_{m}$ (linewidth $4 \mathrm{~nm}$ ), $1.66 \times 10^{-20} \mathrm{~cm}^{2}$ for $E \| N_{g}$ (linewidth 4.1 $\mathrm{nm}$ ), and $2.17 \times 10^{-20} \mathrm{~cm}^{2}$ for $E \| N_{p}$ (linewidth $5.1 \mathrm{~nm}$ ). These values exceed those reported for stoichiometric $\mathrm{YbAG}^{35}$ and are very close to those reported for $\mathrm{KREW}$ ( $R E=\mathrm{Y}$ and $\mathrm{Gd}$ ) with 5 at. \% $\mathrm{Yb}$ doping. ${ }^{11,12}$ The absorption length (1/e level) for $\mathrm{KYbW}$ calculated from the absorption cross section at $981 \mathrm{~nm}$ (room temperature) is only $13.3 \mu \mathrm{m}$ for polarization parallel to the $N_{m}$ principal axis. Such short absorption lengths are particularly useful for the thin disk laser concept.

In order to determine accurately the energy sublevels of ytterbium in this stoichiometric crystal, the optical absorption spectra were measured down to $6 \mathrm{~K}$. Due to the fact that ytterbium has an odd number of electrons in the $4 f$ shell, polarization dependent selection rules for the electronic transitions are not expected. This means that the number and the positions of the absorption peaks have to be independent of the polarization but intensity variation of the peaks associated with the three polarizations is still possible. This could be confirmed experimentally both in the spectra recorded at room temperature and at lower temperatures.

Figure 7 shows the low temperature absorption at $6 \mathrm{~K}$ and the evolution with the temperature for $E \| N_{m}$, all curves measured with $1 \mathrm{~nm}$ spectral resolution and a $50 \mu \mathrm{m}$ thick sample. Since at low temperature the most populated Stark level is the lowest sublevel of the ground state ${ }^{2} F_{7 / 2}(0)$ the bands observed at $6 \mathrm{~K}$ can be associated with transitions from this sublevel to the three Stark levels of the excited state ${ }^{2} F_{5 / 2}$ [denoted for brevity as ${ }^{2} F_{5 / 2}\left(0^{\prime}\right),\left(1^{\prime}\right)$, and $\left(2^{\prime}\right)$ in order of increasing energy].

From the low-temperature absorption spectra we determined the energies of the sublevels of the excited state ${ }^{2} F_{5 / 2}$ : (0') $10188,\left(1^{\prime}\right) 10500,\left(2^{\prime}\right) 10734 \mathrm{~cm}^{-1}$. Two additional lines at 10019 and $9767 \mathrm{~cm}^{-1}$ (see Fig. 7) appear when increasing the temperature which are related to thermal population of the ${ }^{2} F_{7 / 2}(1)$ and the ${ }^{2} F_{7 / 2}(2)$ sublevels, re- spectively, with transitions to ${ }^{2} F_{5 / 2}\left(0^{\prime}\right)$. Again for brevity we denote the ground state levels as ${ }^{2} F_{7 / 2}(0),(1),(2)$, and (3) in order of increasing energy.

A more precise inspection of the low-temperature absorption reveals a certain substructure. This substructure can be related to strongly phonon-electron-coupled transitions. This effect is more pronounced for $R E^{3+}$ ions at the beginning and the end of the lanthanide series. The prevailing role of the Van-Vleck (one-phonon) process in the formation of the phonon sidebands for $R E^{3+}$ ions was outlined previously. ${ }^{36}$ The maximum cross section of the zero line transition at $10188 \mathrm{~cm}^{-1}$ in Fig. 7 drastically decreases when lowering the temperature, though this effect is masked at higher temperatures (where the overall ${ }^{2} F_{7 / 2} \rightarrow{ }^{2} F_{5 / 2}$ oscillator strength seems to remain constant) by population redistribution among the sublevels of the ground state. On the other hand, the most pronounced vibronic features in the $6 \mathrm{~K}$ spectrum which are between 10600 and $10650 \mathrm{~cm}^{-1}$ and near $10710 \mathrm{~cm}^{-1}$ obviously increase their amplitude at lower temperature. This behavior can be attributed to resonant interactions involving the $(0)-\left(0^{\prime}\right)$ transition and some Raman modes $\left(\nu_{4}^{-}, \nu_{4}^{0}, \nu_{4}^{+}\right.$from Table II). Note that additional resonances of other Raman modes from Table II $\left(E_{g}\right.$ and $\left.E_{u}\right)$ with the $(0)-\left(1^{\prime}\right)$ line could also contribute to this effect. Another explanation for the observed substructure near $10710 \mathrm{~cm}^{-1}$ could be, however, the resonance of the Stark sublevel separation of $234 \mathrm{~cm}^{-1}$ between ${ }^{2} F_{5 / 2}\left(1^{\prime}\right)$ and ${ }^{2} F_{5 / 2}\left(2^{\prime}\right)$ with the $239 \mathrm{~cm}^{-1} B_{g}$ phonon from Table II, leading to resonant splitting of the ${ }^{2} F_{5 / 2}\left(2^{\prime}\right)$ sublevel as a result of electron-phonon interaction. ${ }^{37}$ Note that preliminary measurements indicate that such a substructure is absent in low doped KGdW:Yb.

A peculiarity is observed in the behavior of the absorption line at $10019 \mathrm{~cm}^{-1}$ when the temperature reaches $300 \mathrm{~K}$. $\mathrm{Up}$ to $150 \mathrm{~K}$ the intensity of this line increases linearly with temperature but this dependence saturates at higher temperature. This is in controversy with the normal thermal behavior of the absorption process. We attribute the saturation effect observed at $300 \mathrm{~K}$ to resonant interaction between ytterbium ions in the ${ }^{2} F_{7 / 2}(1)$ sublevel (168 $\mathrm{cm}^{-1}$ energy) and the $A_{u}$ vibration mode associated with $\left[\mathrm{WO}_{4}\right]^{2-}$ as reported in Table II.

The increase of the intensity of the zero line at $10188 \mathrm{~cm}^{-1}$ in the $150-200 \mathrm{~K}$ temperature range might be limited by the dynamic range of the spectrophotometer even for a sample thickness as small as $50 \mu \mathrm{m}$. At $6 \mathrm{~K}$ we recorded this line also with an enhanced resolution of $0.5 \mathrm{~nm}$ but still could not resolve it properly measuring a linewidth of $0.66 \mathrm{~nm}$.

\section{B. Fluorescence spectra}

Room- and low-temperature fluorescence spectra were measured in $90^{\circ}$ geometry with excitation by a $200 \mathrm{~mW}$ diode laser at $940 \mathrm{~nm}$ modulated at $1 \mathrm{kHz}$. The fluorescence was dispersed by a $0.46 \mathrm{~m}$ double monochromator (Jobin Yvon - Spex HR 460). A Si photodiode whose spectral response was corrected was used for detection of the infrared emission. It was connected with the lock-in amplifier 


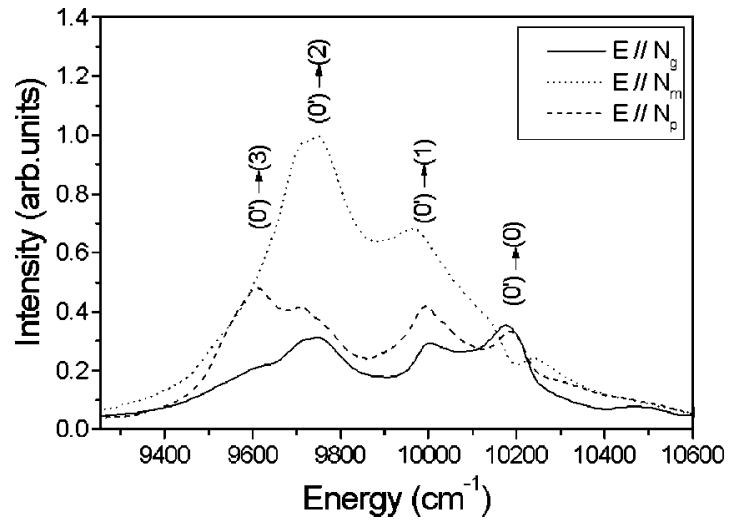

FIG. 8. Polarized emission spectrum of KYbW at room temperature.

(EG\&G, 7265 DSP). The sample cooling for the low temperature measurements was accomplished by the same cryostat with helium-gas flow.

The room-temperature emission spectra are strongly affected by reabsorption. By excitation close to the surface as well as by double recording of the $E|| N_{g}$ spectrum we tried to obtain a comparable plot for all three polarizations which is presented in Fig. 8. The peaks in the measured spectra correspond to the emission from the excited ${ }^{2} F_{5 / 2}$ manifold to the four sublevels of the ground state manifold ${ }^{2} F_{7 / 2}$. The maximum at $10184 \mathrm{~cm}^{-1}\left(10179 \mathrm{~cm}^{-1}\right)$ which appears in the spectra for $E \| N_{p}\left(E \| N_{g}\right)$ is due to the ${ }^{2} F_{5 / 2}\left(0^{\prime}\right)$ $\rightarrow{ }^{2} F_{7 / 2}(0)$ transition. The position of this peak determines the energy of the ${ }^{2} F_{5 / 2}\left(0^{\prime}\right)$ sublevel and it agrees well with the value derived from the $6 \mathrm{~K}$ absorption experiments (10 $188 \mathrm{~cm}^{-1}$ ).

In order to obtain the crystal field splitting of the ground state manifold ${ }^{2} F_{7 / 2}$ low-temperature polarized emission experiments were performed. Figure 9 shows the temperature dependent $E \| N_{m}$ infrared fluorescence spectrum from 6 to $300 \mathrm{~K}$. The spectrum is dominated by three lines at $9633 \mathrm{~cm}^{-1}, 9750 \mathrm{~cm}^{-1}$, and $10020 \mathrm{~cm}^{-1}$ each of them accompanied by phonon added peaks. These three lines correspond to the electronic transitions ${ }^{2} F_{5 / 2}\left(0^{\prime}\right) \rightarrow{ }^{2} F_{7 / 2}(3)$, ${ }^{2} F_{5 / 2}\left(0^{\prime}\right) \rightarrow{ }^{2} F_{7 / 2}(2)$, and ${ }^{2} F_{5 / 2}\left(0^{\prime}\right) \rightarrow{ }^{2} F_{7 / 2}(1)$. The emission line between the ${ }^{2} F_{5 / 2}\left(0^{\prime}\right) \rightarrow{ }^{2} F_{7 / 2}(0)$ sublevels is difficult to observe because of strong reabsorption for this polarization (see Fig. 8).

The Stark sublevels of the ground state manifold ${ }^{2} F_{7 / 2}$ derived from the $6 \mathrm{~K}$ emission spectrum are at $0,168,438$, and $555 \mathrm{~cm}^{-1}$. The crystal field splitting of both manifolds of $\mathrm{KYbW}$, obtained from the low temperature absorption and emission spectra is shown in the inset of Fig. 7.

Having now determined the splitting of the ground state we see that at room temperature only $62 \%$ of the ions are in the ${ }^{2} F_{7 / 2}(0)$ sublevel. This means that the calculation of the maximum absorption cross section from the absorption coefficient should be performed with an $\mathrm{Yb}^{3+}$ concentration of $4 \times 10^{21}$ instead of $6.4 \times 10^{21} \mathrm{~cm}^{-3}$, a deviation which has been overseen in previous literature on KYW or KGdW doped with $\mathrm{Yb}^{3+} .^{8-14}$ Thus for example for $E \| N_{m}$ we obtain a "corrected" maximum cross section of $1.88 \times 10^{-19} \mathrm{~cm}^{2}$ at the zero line $(981 \mathrm{~nm})$.

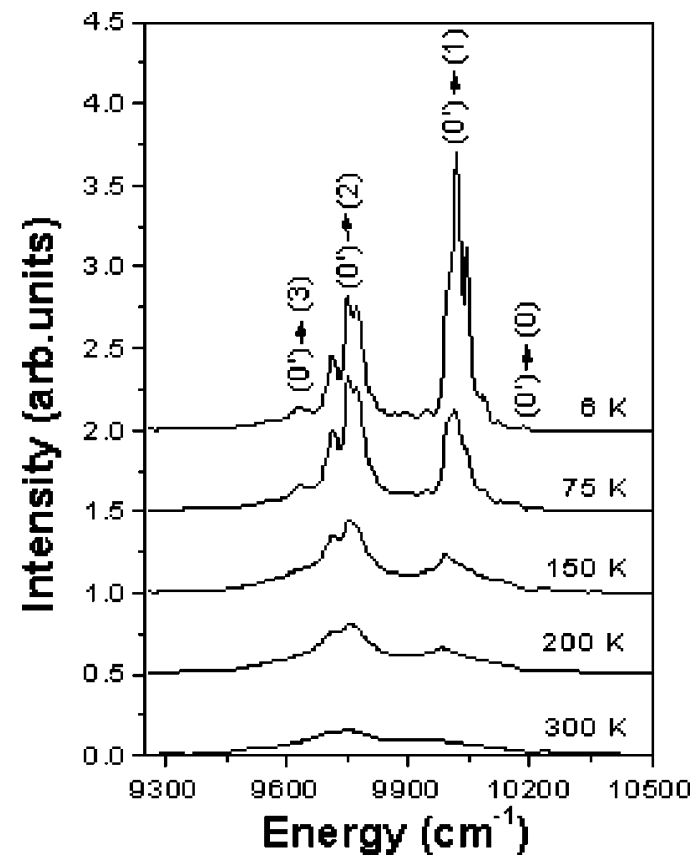

FIG. 9. Temperature evolution of the optical emission of KYbW for $E \| \mid N_{m}$.

Next to the main peaks in the low-temperature spectra in Fig. 9 there are a number of lines, which we assign to vibronic side bands. The main contribution can be again attributed to the zero line transition (10 $188 \mathrm{~cm}^{-1}$ ) coupling with the same phonons outlined in the previous subsection when discussing the low-temperature absorption spectra. A redistribution of the intensity among the electronic and the vibronic lines is observed when changing the temperature. With increasing the temperature the fluorescence spectral profile changes strongly due to the reabsorption effect related to new transitions from thermally populated ground state sublevels and due to the broadening of the individual linewidths. An interesting process is observed in the thermal behavior of the fluorescence peak at $10020 \mathrm{~cm}^{-1}$ which strongly decreases with temperature. The explanation is found after careful analysis of the thermal evolution of the absorption spectra (Fig. 7). One absorption line with vibronic substructure emerges with increasing temperature in the same spectral position. This line is missing in the absorption spectrum at $6 \mathrm{~K}$. Its position and thermal evolution reveal that this absorption line originates from the population of the ${ }^{2} F_{7 / 2}(1)$ sublevel. Obviously, the disappearance of the fluorescence line at $10020 \mathrm{~cm}^{-1}$ is due to the reabsorption effect.

The reciprocity method ${ }^{1,35}$ has been used to calculate the emission cross section using the absorption cross section at room temperature and the splitting determined at low temperature. The calculation is based on the following equation:

$$
\sigma_{e}(\nu)=\sigma_{\mathrm{abs}}(\nu) \frac{Z_{l}}{Z_{u}} \exp \left[\frac{\left(E_{z l}-h \nu\right)}{k T}\right],
$$

where $Z_{u}$ and $Z_{l}$ are the partition functions of the ground and excited states, respectively, and $E_{z l}$ is the temperature- 


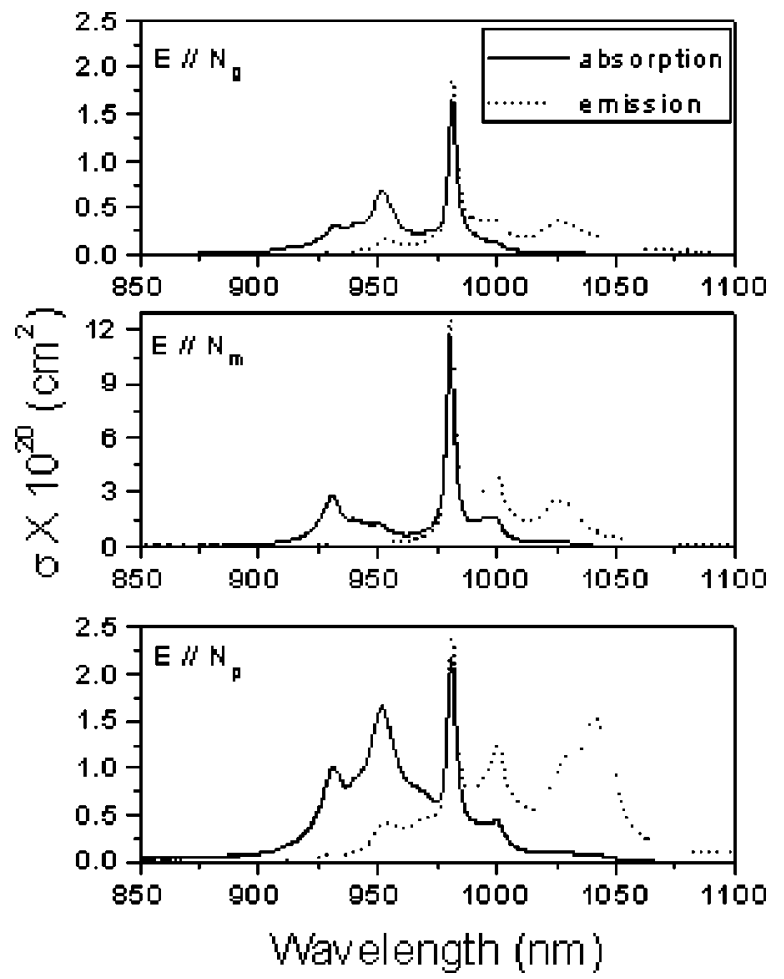

FIG. 10. Emission cross section and absorption cross section of $\mathrm{KYbW}$ at room temperature.

dependent excitation potential. ${ }^{35}$ The calculated ratio $Z_{l} / Z_{u}$ is 1.25 and $E_{z l}$ is equal to $10168.6 \mathrm{~cm}^{-1}$ in our case almost coinciding with the zero-line energy. Figure 10 shows the emission cross sections together with the corresponding absorption cross sections. It can be seen that strong reabsorption should be expected at the maximum emission peak at $981 \mathrm{~nm}$ (zero line).

By averaging $\sigma_{e}(\nu)$ over the three polarizations it is possible to obtain an estimation for the radiative lifetime $\tau_{\text {rad }}$ using the Füchtbauer-Ladenburg equation. ${ }^{1}$ The error originating from the thermal distribution of the population in the ground state manifold ${ }^{2} F_{7 / 2}$ at room temperature when calculating $\sigma_{\mathrm{abs}}(\nu)$ can be suppressed by integrating the corresponding equation over the frequency ${ }^{35}$

$$
\frac{1}{\tau_{\mathrm{rad}}}=8 \pi n^{2} \int \frac{\left\langle\sigma_{e}(\nu)\right\rangle}{\lambda^{2}} d \nu
$$

where \langle\rangle denotes averaging over the polarization. Using the $\sigma_{e}(\nu)$ dependence as calculated with the reciprocity method (Fig. 10) we arrive thus at $\tau_{\text {rad }}=270 \mu$ s at room temperature.

\section{Lifetime measurements}

The excitation of the samples for the fluorescence lifetime measurements was at $940 \mathrm{~nm}$ by a tunable optical parametric oscillator (OPO, VEGA BMI) which is pumped by the third harmonic of a $Q$-switched Nd:YAG laser (SAGA $120 \mathrm{BMI}$ ). The OPO's linewidth amounts to $0.2 \mathrm{~cm}^{-1}$ with a pulse duration of $\sim 5$ ns. The energy of the idler pulse delivered by the OPO at $940 \mathrm{~nm}$ is up to $60 \mathrm{~mJ}$. The fluorescence was collected with a $f=6 \mathrm{~cm}$ lens and dispersed with an Oriel $1 / 8 \mathrm{~m}$ single monochromator at $90^{\circ}$ geometry. The detection was with an InGaAs photodiode connected to a preamplifier. Decay curves were recorded using a 54616 Hewlett Packard oscilloscope with data acquisition on a computer. The measurements were carried out at room temperature.

The accurate measurement of the excited-state lifetime is important as a further step for the characterization of $\mathrm{KYbW}$ as a laser material. It is well known that radiation trapping and total internal reflection (TIR) can strongly affect the measured lifetimes. This problem is strongly pronounced in $\mathrm{KYbW}$, which is characterized by a large degree of overlap between absorption and emission. As a term radiation trapping is commonly referred to the effect of reabsorption of the initial emission by ions in the ground state. This reabsorption is followed by reemission and results in lengthening of the measured lifetime. In order to eliminate this effect optically thin samples should be used for lifetime measurements. TIR, which is typical for solids because of their higher refractive indices, further increases the probability for reabsorption of the emitted photons by ions in the ground state. For KYbW with an average refractive index of $n=2.04$ at $1000 \mathrm{~nm}$ all photons directed to the crystal-air interface at an angle greater than $30^{\circ}$ will be reflected. Thus, even in optically thin samples, TIR produces an increase of the emission pathlength in the crystal and causes an artificial lengthening of the observed lifetimes. Currently the problem of eliminating these effects is widely discussed in the literature ${ }^{38,39}$ and different methodologies are proposed for its solution.

In the present work we attempted to eliminate radiation trapping and TIR using fine powder of KYbW with 25-60 $\mu \mathrm{m}$ diameter of the particles and ethylene-glycol (EG) for refractive-index matching. (In principle the use of an indexmatching fluid with a refractive index $n \sim 2$ should be better but index-matching fluids with $n$ higher than 1.8 are toxic and that is why we chose EG with $n=1.52$ ).

Five samples were prepared: one pure powder sample placed in a quartz cuvette and four samples with different concentration of the KYbW powder, immersed in EG, and placed in similar quartz cuvettes. Also, a measurement of the time decay of the fluorescence in a KYbW crystal with thickness of $170 \mu \mathrm{m}$ was carried out for comparison.

The fluorescence lifetime curves for all samples are characterized by a single exponential decay behavior. The lifetime measured for the $170 \mu \mathrm{m}$ crystal was $600 \mu$ s while for the powder sample (without EG) it amounted to $320 \mu \mathrm{s}$. The particle size of the powder is of the order of the absorption length and the large difference between these two measurements can be explained only by the radiation trapping in the $170 \mu \mathrm{m}$ crystal, because TIR takes place in both cases.

All powder samples, which were immersed in EG show significant reduction of the observed lifetimes in comparison with the pure powder sample. The measured lifetimes decrease with decreasing the concentration of the powder in EG. Decreasing the concentration for samples with a refractive-index-matching fluid significantly reduces the average pathlength for the emission in the sample, thus decreasing the probability for reabsorption due to TIR. There is one minimal concentration, below which no further change 
TABLE III. Lifetime of the infrared fluorescence at $1040 \mathrm{~nm}$ of KYbW powder immersed in ethylene-glycol versus concentration (weight concentration $=$ weight of powder/weight of powder $+E G)$.

\begin{tabular}{cc}
\hline \hline Weight concentration $(\%)$ & Lifetime $(\mu \mathrm{s})$ \\
\hline 0.23 & 200 \\
0.52 & 200 \\
1.92 & 250 \\
2.92 & 270 \\
100 & 350 \\
\hline
\end{tabular}

of the lifetime could be observed. This limit for the lifetime, measured for diluted samples, is $200 \mu \mathrm{s}$. All results are presented in Table III.

The shortest lifetime of $200 \mu$ s in diluted samples suggests efficient elimination of TIR and radiation trapping. It makes us confident that it is close to the intrinsic single-ion lifetime. Our value is closer but still below the recently measured $300 \mu \mathrm{s}$ for KYW crystals with low doping level of $\mathrm{Yb}^{3+}+40$

The ratio of the fluorescence and radiative lifetimes provides a direct estimation of $74 \%$ for the intrinsic quantum efficiency. As in the case of stoichiometric YbAG (Ref. 35) improved material development is expected to increase the fluorescence lifetime and the quantum efficiency by reduction of the excitation quenching caused by impurities in the $\mathrm{KYbW}$ lattice. At present such heating effects seem to predominate over those resulting from the quantum defect.

\section{LASER EXPERIMENTS}

The laser experiments with KYbW were performed with a V-type astigmatically compensated resonator with a length of $57 \mathrm{~cm}$. The $0.5 \mathrm{~mm}$ thin $\mathrm{KYbW}$ sample was positioned in the focal region between the folding and rear mirrors which were both highly reflecting in the emission spectral range and with $10 \mathrm{~cm}$ radius of curvature. The output coupler in the other end of the cavity was a plane mirror with a transmission (if not otherwise specified) of $1 \%$. The thin plane of $\mathrm{KYbW}$ was cut and oriented for propagation along the $\boldsymbol{b}\left(N_{p}\right)$ axis and polarization along the $N_{m}$ crystallo-optic axis. The polarization choice plays a very important role because it guarantees maximum emission cross section and amplification. At the given thickness, however, the absorption for the pump radiation which is also polarized parallel to $N_{m}$ is unnecessarily large and to avoid reabsorption at the laser wavelength in the unpumped rear side of the sample one has to choose pump wavelengths away from the maximum of the absorption cross section.

The pump source was a homemade CW Ti:sapphire laser optimized for operation at relatively long wavelengths as the one used previously by us. ${ }^{41}$ It was tunable between 900 and $1000 \mathrm{~nm}$ when pumped by $24 \mathrm{~W}$ (all lines) of an argon ion laser (Spectra Physics 2040) and had a maximum output power of about $3 \mathrm{~W}$ between 920 and $970 \mathrm{~nm}$. The pump beam was focussed by a $f=6.3 \mathrm{~cm}$ lens through the folding mirror of the $\mathrm{KYbW}$ laser cavity which was transmitting for the pump radiation but not specially designed for that pur-

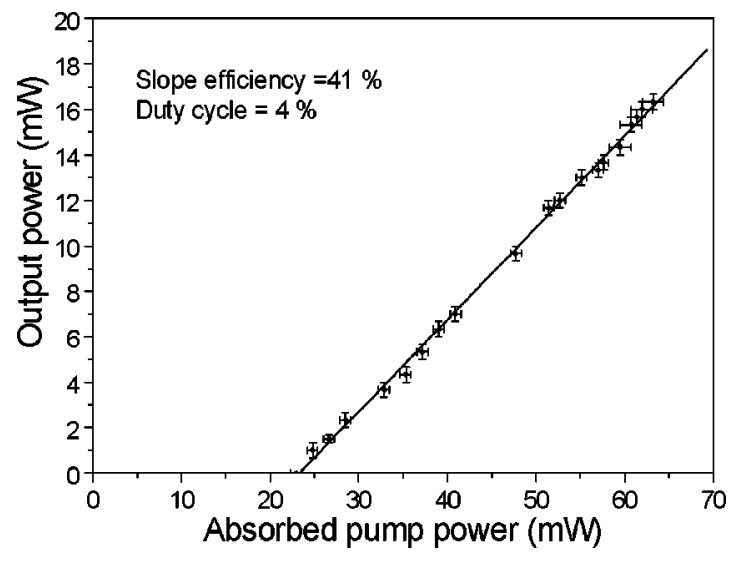

FIG. 11. Average output power of the quasi-CW KYbW laser versus average absorbed pump power.

pose. The uncoated KYbW sample was inclided under Brewster angle to minimize the Fresnel losses but this did not result in essential modification of the polarization and propagation directions inside the sample. No special care was taken for good thermal contact or cooling of the sample, hence in order to reduce the thermal load we had to employ a chopper with a duty cycle of $4 \%$ which reduced the average powers 25 times. All average powers given in this section are those obtained with this chopper.

Figure 11 shows the output power of the KYbW laser measured at $1074 \mathrm{~nm}$ as a function of the absorbed pump power for a pump wavelength of $963 \mathrm{~nm}$. This pump wavelength is situated in the dip of the absorption curve in Fig. 6 and has been chosen because it is close to the wavelengths where high power InGaAs laser diodes are available, too. At this pump wavelength the sample absorbed about $92 \%$ of the pump radiation which is in good agreement with the transmission (10\%) calculated for $E \| N_{m}$ from Fig. 6 if we take into account the effective crystal length under Brewster angle. The pump pulse duration used in Fig. 11 was $350 \mu \mathrm{s}$ corresponding to a chopper frequency of about $110 \mathrm{~Hz}$. At this chopper frequency and pump pulse duration no saturation in the dependence depicted in Fig. 11 is observed and the maximum output power obtained $(16 \mathrm{~mW})$ corresponds to a conversion efficiency of $25 \%$ with respect to the absorbed pump power. The slope efficiency with respect to the absorbed power estimated from the Fig. 11 is $41 \%$ and exceeds the slope efficiency recently reported $(27 \%)$ in a similar quasi-CW regime with stoichiometric YbAG. ${ }^{35}$ Note that the free running wavelength obtained by us was $1074 \mathrm{~nm}$ which is far from the maximum of the fluorescence curve and can be explained by reabsorption effects at shorter wavelengths.

The dependence in Fig. 11 was recorded for pump pulse durations sufficiently short to avoid the onset of thermal effects during one pump period. This can be seen from Fig. 12 where we have plotted the dependence of the average output power versus pump pulse duration which was varied by changing the chopper frequency at a constant value of the average absorbed pump power $(62 \mathrm{~mW})$. Slightly above $400 \mu$ s degradation of the laser performance is observed but we believe such thermal effects could be managed by special 


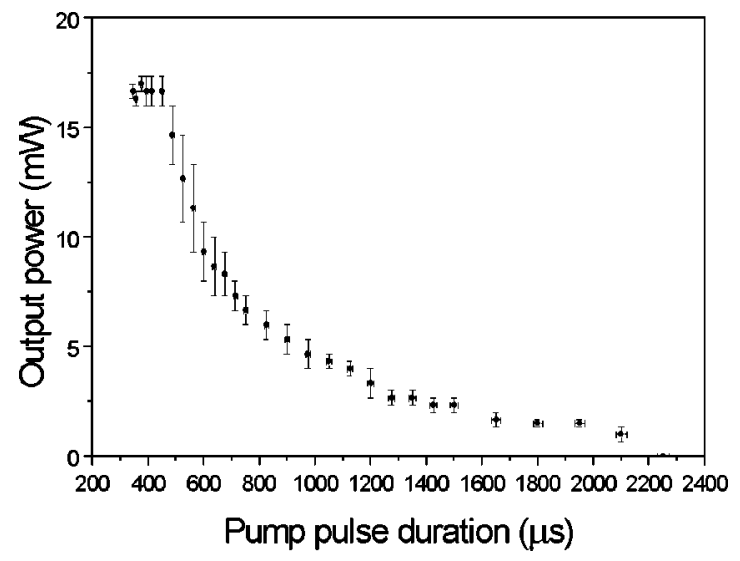

FIG. 12. Average output power of the quasi-CW KYbW laser versus pump pulse duration.

mount and cooling systems for the KYbW sample.

Also the temporal shape of the output pulse generated depends on the pump pulse duration. Figure 13 shows two records obtained for pump pulse durations of 350 and $1500 \mu \mathrm{s}$. It can be seen that at longer pump pulse durations oscillation ceases before the end of the pump pulse. The uninterrupted generation of the $\mathrm{KYbW}$ laser with long pulse pumping is almost as long as the generation pulse observed with short pulse pumping. The absorbed pump pulse energy amounted to $0.55 \mathrm{~mJ}$ in the case of short pulse pumping (chopper frequency equal to $110 \mathrm{~Hz}$ ) and to $2.5 \mathrm{~mJ}$ in the case of long pulse pumping (chopper frequency equal to 25 $\mathrm{Hz})$. The output energy was almost the same $(0.1 \mathrm{~mJ})$ in both cases but the output power is roughly 4 times higher in the case of short pulse pumping.

The $1 \%$ output coupling used was optimum for this laser. The output power dropped to $60 \%$ of its maximum value when using an output coupler with less $(0.6 \%)$ transmission and to $30 \%$ for an output coupler with higher $(1.9 \%)$ transmission.

Finally, we note that similar results in terms of conversion efficiency and output power were obtained also when pumping in the wing of the absorption curves near $922 \mathrm{~nm}$. On the other hand, the longest pump wavelength that still permitted to reach the threshold for laser generation at the given crystal thickness was $974 \mathrm{~nm}$.

\section{CONCLUSION}

In conclusion, the method of TSSG slow-cooling permitted the growth of inclusion-free KYbW single crystals with laser quality. $\mathrm{KYbW}$ is a monoclinic biaxial crystal with the space group $C 2 / c$ (point group $2 / m$ ) which is transparent between 350 and $5000 \mathrm{~nm}$. Its unit cell parameters are $a=10.590(4) \AA, b=10.290(6) \AA, c=7.478(2) \AA, \beta$ $=130.70(2)^{\circ}$. The two principal axes $N_{g}$ and $N_{m}$ of the optical indicatrix are located in the $\boldsymbol{a}-\boldsymbol{c}$ plane and the other principal axis $N_{p}$ is parallel to $\boldsymbol{b}[010]$. The principal axis with maximum refractive index $N_{g}$ is rotated by $19^{\circ}$ from the $\boldsymbol{c}$ axis. Sellmeier coefficients for KYbW have been deter-

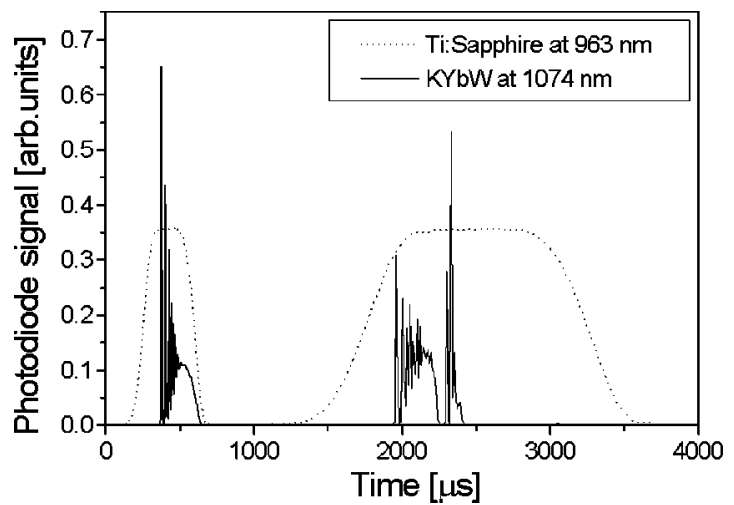

FIG. 13. Temporal profiles of the pump (dashed line) and laser (solid line) pulses for short (left) and long (right) pulse pumping.

mined for the visible and near-IR spectral range at room temperature. The thermo-optic coefficients have also been measured at room temperature.

The highest value of the absorption cross section at room temperature is obtained at $10195 \mathrm{~cm}^{-1}(981 \mathrm{~nm}$, zero line) for polarization parallel to the $N_{m}$ axis: it amounts to 1.88 $\times 10^{-19} \mathrm{~cm}^{2}$. The resonant absorption in this spectral region is due to ytterbium transitions between the only two states ${ }^{2} F_{7 / 2}$ and ${ }^{2} F_{5 / 2}$. These two manifolds have been resolved by absorption and emission measurements at room and low temperature. Raman spectroscopy revealed the phonon energies in $\mathrm{KYbW}$ and this information was used to explain the vibronic substructure of the absorption spectrum. Fluorescence decay measurements provided a reliable value of $200 \mu$ s for the intrinsic upper level lifetime and $74 \%$ for the intrinsic quantum efficiency at room temperature.

Finally, for the first time to our knowledge, we obtained laser operation with stoichiometric $\mathrm{KYbW}$. The slope efficiency exceeded that demonstrated previously with $\mathrm{YbAG}$ which is the only other known stoichiometric $\mathrm{Yb}$ laser. The $\mathrm{KYbW}$ laser was studied only in the quasi-CW regime because of the onset of thermal effects in the absence of crystal cooling and at room temperature. It operated at $1074 \mathrm{~nm}$, far from the fluorescence maximum and could be pumped at wavelengths as long as $974 \mathrm{~nm}$, which means that laser diode pumping is realistic. Even better results are expected by pumping yet thinner samples of KYbW with improved crystal quality at longer wavelengths in which case reduction of the heat to be dissipated is expected. Moreover $\mathrm{KYbW}$ should have better thermal conductivity than the well known hosts KYW and KGdW. This makes us confident that real $\mathrm{CW}$ operation of KYbW with laser diode pumping could be achieved in the near future.

\section{ACKNOWLEDGMENTS}

We are grateful to Dr. Todor Kirilov for helpful discussions. This work was supported by CICyT under Projects No. MAT99-1077-C02 and 2FD97-0912-C02, by CIRIT under Project No. 1999SGR 00183, by Acciones Integradas Hispano-Alemanas, and by the Large Scale Laser Facility program of EU for MBI. 
*Permanent address: University of Chemical Technology and Metallurgy, 1756 Sofia, Bulgaria.

†Author to whom correspondence should be addressed.

${ }^{1}$ L.D. Deloach, S.A. Payne, L.L. Chase, L.K. Smith, W.L. Kway, and W.F. Krupke, IEEE J. Quantum Electron. 29, 1179 (1993).

${ }^{2}$ X. Zou and H. Toratani, Phys. Rev. B 52, 15889 (1995).

${ }^{3}$ W.F. Krupke, IEEE J. Sel. Top. Quantum Electron. 6, 1287 (2000)

${ }^{4}$ T.T. Basiev, A.A. Sobol, P.G. Zverev, V.V. Osiko, and R.C. Powell, Appl. Opt. 38, 594 (1999).

${ }^{5}$ P.V. Klevtsov and L.P. Kozeeva, Dokl. Akad. Nauk SSSR 185, 571 (1969) [Sov. Phys. Dokl. 14, 185 (1969)].

${ }^{6}$ A.A. Kaminskii, A.A. Pavlyuk, T.I. Butaeva, L.I. Bobovich, and V.V. Lyubchenko, Izv. Akad. Nauk SSSR, Neorgan. Mate. 15, 541 (1979) [Inorg. Mater. (Transl. of Neorg. Mater.) 15, 424 (1979)].

${ }^{7}$ A. Majchrowski, M.T. Borowiec, E. Michalski, J. Zmija, V. Dyakonov, H. Szymczak, T. Zayarnyuk, and M. Baranski, Cryst. Res. Technol. 36, 283 (2001).

${ }^{8}$ A. Brenier and G. Boulon, J. Alloys J. Alloys Compd. 323-324, 210 (2001).

${ }^{9}$ A. Brenier, J. Lumin. 92, 199 (2001).

${ }^{10}$ N.V. Kuleshov, A.A. Lagatsky, V.G. Shcherbitsky, V.P. Mikhailov, E. Heumann, T. Jensen, A. Diening, and G. Huber, Appl. Phys. B: Lasers Opt. 64, 409 (1997).

${ }^{11}$ A.A. Lagatsky, N.V. Kuleshov, and V.P. Mikhailov, Opt. Commun. 165, 71 (1999).

${ }^{12}$ N.V. Kuleshov, A.A. Lagatsky, A.V. Podlipensky, V.P. Mikhailov, and G. Huber, Opt. Lett. 22, 1317 (1997).

${ }^{13}$ G. Metrat, M. Boudeulle, N. Muhlstein, A. Brenier, and G. Boulon, J. Cryst. Growth 197, 883 (1999).

${ }^{14}$ A. Brenier, G. Metrat, N. Muhlstein, F. Bourgeous, and G. Boulon, Opt. Mater. 16, 189 (2001).

${ }^{15}$ F. Brunner, G.J. Spühler, J. Aus der Au, L. Krainer, F. MorierGenoud, R. Paschotta, N. Lichtenstein, S. Weiss, C. Harder, A.A. Lagatsky, A. Abdolvand, N.V. Kuleshov, and U. Keller, Opt. Lett. 25, 1119 (2000).

${ }^{16}$ P. Klopp, V. Petrov, U. Griebner, and G. Erbert, Opt. Express 10, 108 (2002).

${ }^{17}$ H. Liu, J. Nees, and G. Mourou, Opt. Lett. 26, 1723 (2001).

${ }^{18}$ H. Liu, J. Nees, and G. Mourou (unpublished).

${ }^{19}$ J. Aus der Au, G.J. Spühler, T. Südmeyer, R. Paschotta, R. Hövel, M. Moser, S. Erhard, M. Karszewski, A. Giesen, and U. Keller, Opt. Lett. 25, 859 (2000).

${ }^{20}$ S. Erhard, J. Gao, A. Giesen, K. Contag, A. A. Lagatsky, A. Abdolvand, N. V. Kuleshov, J. Aus der Au, G. J. Spühler, F. Brunner, R. Paschotta, and U. Keller (unpublished).
${ }^{21}$ R. Solé, V. Nikolov, X. Ruiz, J. Gavaldà, X. Solans, M. Aguiló, and F. Diaz, J. Cryst. Growth 169, 600 (1996).

${ }^{22}$ M.C. Pujol, R. Solé, Jna. Gavaldà, J. Massons, M. Aguiló, F. Diaz, V. Nikolov, and C. Zaldo, J. Mater. Res. 14, 3739 (1999).

${ }^{23}$ M.C. Pujol, X. Mateos, R. Solé, J. Massons, Jna. Gavaldà, X. Solans, F. Díaz and M. Aguiló, J. Appl. Crystallogr. 35, 108 (2002).

${ }^{24}$ G. M. Sheldrick, SHELXS97. A Computer Program for Crystal Structure Determination, 1997, University of Göttingen, Germany.

${ }^{25}$ G. M. Sheldrick, SHELXL97. A Computer Program for Crystal Structure Determination, 1997, University of Göttingen, Germany.

${ }^{26}$ R. Solé, V. Nikolov, A. Vilalta, J. J. Carvajal, J. Massons, Jna. Gavaldà, M. Aguiló, and F. Díaz. J. Mater Res. 17 (2002).

${ }^{27}$ M. Born and E. Wolf, Principles of Optics. Electromagnetic Theory of Propagation, Interference and Diffraction of Light, 6th ed. (Pergamon, Oxford, 1993).

${ }^{28}$ J. Hanuza and L. Macalik, Spectrochim. Acta, Part A 43, 361 (1987).

${ }^{29}$ L. Macalik, P.J. Deren, J. Hanuza, W. Strek, A.A. Demidovich, and A.N. Kuzmin, J. Mol. Struct. 450, 179 (1998).

${ }^{30}$ S.P.S. Porto and J.F. Scott, Phys. Rev. 157, 716 (1967).

${ }^{31}$ L.A. Woodward, and H.L. Roberts, Trans. Faraday Soc. 52, 615 (1956).

${ }^{32}$ L. Macalik, J. Hanuza, and A.A. Kaminskii, J. Mol. Struct. 555, 289 (2000).

${ }^{33}$ A.A. Lagatsky, A. Abdolvand, and N.V. Kuleshov, Opt. Lett. 25, 616 (2000).

${ }^{34}$ J.K. Viscakas, I.V. Mochalov, A.V. Mikhailov, R.F. Klevtsova, and A.V. Lyubimov, Litov. Fiz. Sbornik 28, 224 (1988) [Sov. Phys. Collect. 28, 64 (1988)].

${ }^{35}$ F.D. Patel, E.C. Honea, J. Speth, S.A. Payne, R. Hutcheson, and R. Equal, IEEE J. Quantum Electron. 37, 135 (2001).

${ }^{36}$ V. V. Ovsyankin, in Spectroscopy of Solids Containing Rare Earth Ions, edited by A. A. Kaplyanskii and R. M. Macfarlane (Elsevier, Amsterdam, 1987), p. 343.

${ }^{37}$ A. Lupei, V. Lupei, V.N. Enaki, C. Presura, and A. Petraru, Spectrochim. Acta, Part A 55, 773 (1999).

${ }^{38}$ D.S. Sumida and T.Y. Fan, Opt. Lett. 19, 1343 (1994).

${ }^{39}$ M.P. Hehlen, J. Opt. Soc. Am. B 14, 1312 (1997).

${ }^{40}$ A.A. Demidovich, A.N. Kuzmin, G.I. Ryabtsev, M.B. Danailov, W. Strek, and A.N. Titov, J. Alloys Compd. 300-301, 238 (2000).

${ }^{41}$ V. Petrov, U. Griebner, D. Ehrt, and W. Seeber, Opt. Lett. 22, 408 (1997). 